\title{
Experimental Demonstration of Rate-adaptive Concatenated Codes for Flexible Optical Networks
}

Mehmood, Tayyab; Yankov, Metodi P.; Hansen, Henrik E.; Forchhammer, Søren

Published in:

IEEE Photonics Technology Letters

Link to article, DOI:

10.1109/LPT.2021.3127656

Publication date:

2021

Document Version

Peer reviewed version

Link back to DTU Orbit

Citation $(A P A)$ :

Mehmood, T., Yankov, M. P., Hansen, H. E., \& Forchhammer, S. (2021). Experimental Demonstration of Rateadaptive Concatenated Codes for Flexible Optical Networks. IEEE Photonics Technology Letters, 33(24), 14471450. https://doi.org/10.1109/LPT.2021.3127656

\section{General rights}

Copyright and moral rights for the publications made accessible in the public portal are retained by the authors and/or other copyright owners and it is a condition of accessing publications that users recognise and abide by the legal requirements associated with these rights.

- Users may download and print one copy of any publication from the public portal for the purpose of private study or research.

- You may not further distribute the material or use it for any profit-making activity or commercial gain

- You may freely distribute the URL identifying the publication in the public portal 


\title{
Experimental Demonstration of Rate-adaptive Concatenated Codes for Flexible Optical Networks
}

\author{
Tayyab Mehmood, Graduate Student Member, IEEE, Metodi P. Yankov, Member, IEEE, \\ Henrik E. Hansen and Søren Forchhammer, Member, IEEE
}

\begin{abstract}
We experimentally demonstrate a rate-adaptive concatenated forward error correction (FEC) scheme to minimize the information rate loss due to varying channel conditions. The concatenated coding scheme is based on inner soft-decision polar code and outer hard-decision staircase code. An experiment in a wavelength-division multiplexed (WDM) system with different SNR per WDM channel is performed by employing concatenated codes with various coded modulation schemes over a target distance of $140 \mathrm{~km}$ which is relevant for extending the reach of data center interconnects. Experimental results show that the achieved data rate of the WDM system can be improved up to $37.6 \%$ with the proposed coding scheme w.r.t. the fixed code rate ZR FEC.
\end{abstract}

Index Terms-Polar codes, staircase codes, multilevel coding, rate flexibility, 400ZR FEC, coherent transceivers, data center interconnects.

\section{INTRODUCTION}

$\mathbf{T}$ HE rapid increase in bandwidth-hungry internet applications has led to constant pressure on optical transport networks (OTNs). To avoid the anticipated capacity bottleneck, the physical layer architecture of future OTNs must incorporate novel coded modulation (CM) schemes that can be optimized in terms of spectral efficiency (SE), latency, and power consumption [1]. In particular, novel forward error correction (FEC) techniques have to be put forward for coherent transceivers as a solution to operate at very high throughput and utilize FEC decoders of lower complexity.

Concatenated FEC (CFEC) is an efficient technique for optical communications, where high reliability as well as high data rate is required. In particular, a serial concatenation of outer hard-decision (HD) and inner soft-decision (SD) codes is gaining traction due to complexity reduced FEC decoders [2][7]. For a CFEC scheme, the design of inner SD codes plays an important role, since an SD decoder generally dissipates more power relative to their HD counterparts [8].

The optical inter-networking forum proposed the 400ZR coding scheme for in-region data center interconnects (DCIs) [9], where the high performance yet low power-consuming concatenated Hamming-staircase code is adopted [10]. In [11], the error-floor performance of the 400ZR FEC algorithm was investigated by using FPGAs.

Future OTNs demand transceivers to function at different signal-to-noise ratios (SNRs) requiring rate adaptivity. The

T. Mehmood, M. P. Yankov, H. E. Hansen and S. Forchhammer are with the Department of Photonics Engineering, Technical University of Denmark, 2800 Kongens Lyngby, Denmark (e-mail: tayy@fotonik.dtu.dk; meya@fotonik.dtu.dk; heeha@fotonik.dtu.dk; sofo@fotonik.dtu.dk). current standard 400ZR FEC does not offer this but for the future extensions of 400ZR (ZR+), it can either be achieved by shortening or puncturing of inner or outer code, e.g. shortening the staircase codes [12] or by utilizing a set of FEC codes with different rates [13]. Shortening of the staircase codes comes at the cost of performance degradation [12], while a set of FEC code designs implies an increased chip area. Hence, for future extensions of the current standard [9], it is highly desirable to have a low-power, high-performance, and/or rate-adaptive CFEC solution with an error-floor below $10^{-15}$.

To offer rate-adaptivity with fine granularity, a bitinterleaved coded modulation (BICM) based CFEC solution was proposed in [5], where a performance optimized and rate-adaptive inner polar code is employed. More recently, we proposed a concatenated multilevel coding scheme [7] having rate-adaptivity with two degrees of freedom with fine granularity which offers better complexity and performance compared to the BICM scheme of [5].

In this paper, we experimentally demonstrate a fully loaded wavelength division multiplexed (WDM) system over $140 \mathrm{~km}$ (typical target distance for ZR+ [14]) with varying SNR per channel. Error-free transmission with significantly improved achieved data rate w.r.t the fixed-rate FEC of [9] is experimentally demonstrated by employing the concatenated coding scheme with a binary reflected Gray labeled 64 quadrature amplitude modulation (QAM).

To our knowledge, this is the first time a concatenated coding scheme with outer HD and inner SD FEC having rate flexibility (with one and two degrees of freedom with nearcontinuous granularity) is experimentally demonstrated over the optical fiber link.

\section{Rate-ADAPtive Concatenated Codes}

The proposed parallel decoding based MLC scheme illustrated in Fig. 1.a comprises an outer HD staircase code and an inner SD polar code. The job of the inner SD code is to bring the bit-error rate (BER) down to the decoding threshold of an outer HD code, while the outer code is responsible both for correcting the remaining errors and achieving output BER below $10^{-15}$. We consider a BER threshold $p$ of the outer staircase code of [15] to calculate the performance of the CM system, as illustrated in Fig. 1.a.

The performance-optimized and rate adaptive inner $\left(N_{p c}, K_{p c}\right)$ polar code is obtained by choosing a single frozen set at which the system performance over the range of data rates is relatively constant. The frozen set is designed according to the desired BER threshold $p$ of the outer code, 




Fig. 1. (a) Block diagram of the experimental testbed. (b) Received SNR (dB) profile for the studied concatenated coding scheme as a function of the channel given by external cavity laser (ECL) index.

as specified in [7]. After fixing the CFEC parameters, rateadaptivity can be achieved by tuning the number of inner code information bits, $K_{p c}$.

The proposed approach consists of an outer staircase code with extended $\mathrm{BCH}$ component codes. In the encoding process, the information bits are encoded and the generated parity bits are arranged into a staircase block. The resultant outer encoded bits are first interleaved by the interleaver $\pi_{1}$ and then de-multiplexed into $T_{M L C}=\left(J \times D^{2}\right) /\left(K_{p c}+L_{0}\right)$ sub-blocks [7], where $J, D^{2}$, and $L_{0}$ are the total number of staircase blocks transmitted, size of each staircase block, and the number of bits that bypass the inner SD code, respectively. Each sub-block is of size $K_{p c}+L_{0}$ and the number of $L_{0}$ and/or $K_{p c}$ bits can be tuned at the de-multiplexer (DEMUX) to (i) achieve rate adaptivity with two degrees of freedom, (ii) control the amount of inner SD decoding operations and (iii) simultaneously switch between BICM $\left(L_{0}=0\right)$ and MLC $\left(L_{0} \neq 0\right)$. Typically, $K_{p c}+L_{0} \ll D^{2}$, resulting in multiple SD codewords per staircase block, as illustrated in Fig. 1.a. The rate per $M$-QAM symbol of the proposed MLC scheme can be calculated as:

$$
R_{M L C}=\left(\left(\frac{K_{p c}+L_{0}}{N_{p c}+L_{0}}\right) \times R_{s c}\right) \times \log _{2}(M),
$$

where, $R_{s c}=239 / 255$ is the rate of the outer staircase code. For $L_{0}=0$, the rate $R_{B I C M}$ of BICM-based concatenated coding scheme is equal to $R_{M L C}$ of Eq. (1). The transmitter with BICM-based polar-staircase code and 16QAM is inline with the 400ZR FEC but with different inner SD code. The absolute complexity of the inner decoder is not evaluated in this letter; rather, the relative complexity savings are considered, which are the same regardless of the decoder's complexity and stem from the fewer encoding/decoding operations due to the bypassed bits. For a fixed transmission rate, the relative complexity-reduction $T_{r e d}(\%)$ in SD decodings achieved by employing the MLC scheme w.r.t BICM can by calculated as [7, Eq. (9)]:

$$
T_{\text {red }}=100 \times\left(1-\frac{K_{p c}}{K_{p c}+L_{0}}\right) .
$$

Data on the branch $L_{0}$ bypass the inner SD FEC and are assigned to the high reliability bit levels of the QAM symbols. The cyclic redundancy check (CRC) of length 6 bits is applied on the $K_{p c}$ information bits and the resultant concatenated block is encoded by the systematic polar encoder. In this letter, the code length $N_{p c}=1024$ bits is considered. The inner encoded bits are first bit-interleaved by the interleaver $\pi_{2}$ and then assigned to the remaining bit levels of the QAM symbols.

At the receiver, pilot-based DSP is applied with a pilot rate of $10 \%$ and the Gaussian auxiliary channel is used for demapping [16]. Hard-decisions are performed on the mostreliable $L_{0}$ bits to estimate the $\hat{L}_{0}$ bits. The log-likelihood ratios estimated for the remaining $\hat{N}_{p c}$ bits are first deinterleaved by $\pi_{2}^{-1}$ and then decoded using a CRC-aided successive-cancellation list decoder with a list size of 8 . The inner decoder outputs HD data blocks of length $\hat{K}_{p c}$ bits. The HD data-streams of both $\hat{L}_{0}$ and $\hat{K}_{p c}$ are multiplexed and fed to the outer de-interleaver $\pi_{1}^{-1}$.

In this letter, the outer code is treated as fixed and is not considered in the simulation. Without loss of generality, the performance of both of the MLC- and BICM-based CM systems is estimated in terms of BER out of the multiplexer (MUX), as depicted in Fig. 2. The BER is then a strong indicator of the HD decoder's performance and thus the overall CFEC system performance [17]. In the experiment, we consider the target data rate "achieved" if the BER at the MUX is below the threshold $p$. The validity of this assumption is further discussed below. For the concatenated polar-staircase code design, we consider an ITU-T G.709.2 [15, Table A.1] recommended staircase code as the outer code. The staircase code has a threshold $p=4.5 \times 10^{-3}$ which gives BER below $10^{-15}$ as specified in G.907.2 and which we refer to as error-free. However, to enable a practical implementation with penalty due to imperfect interleaving, we leave a margin of $10 \%$ and set threshold $p$ to $4.0 \times 10^{-3}$ and evaluate this in Sec. IV. Satisfying this threshold, we refer to as being errorfree. 


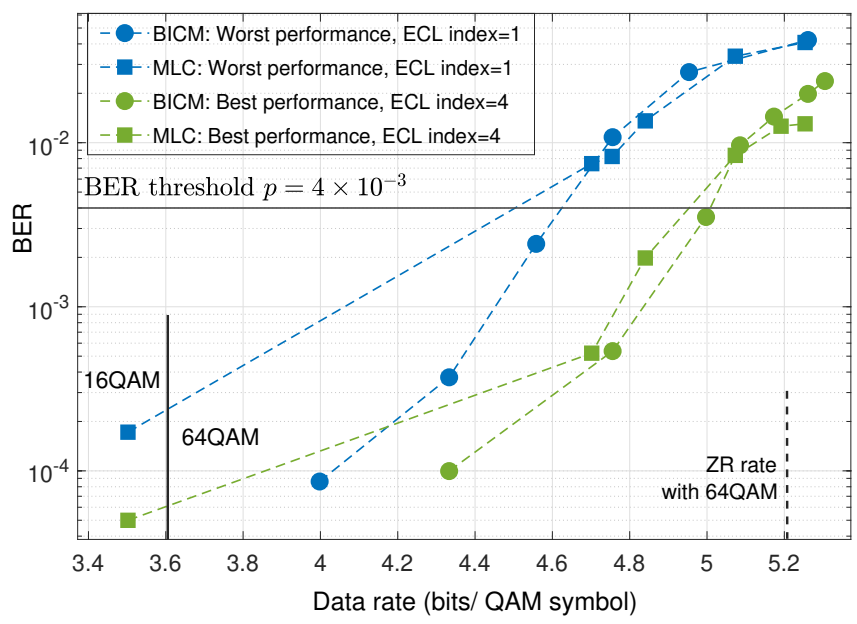

Fig. 2. WDM transmission: Output BER performance of coded modulation schemes with the best and worst ECL channels as a function of data rate.

\section{EXPERIMENTAL SETUP}

The experimental testbed demonstrating rate-adaptive concatenated codes for optical transmission is shown in Fig. 1.a. The CM symbols are pulse-shaped with a square-root raised cosine filter of roll-off 0.01 and sent to an arbitrary waveform generator (AWG) at a sampling rate of $64 \mathrm{GSa} / \mathrm{s}$. A 40channel, $100 \mathrm{GHz}$ spaced, C-band WDM system is considered using $2 \mathrm{I} / \mathrm{Q}$ modulators at 32 GBaud with independent data on each: 1 modulator for the 8 channels of interest carried by external cavity lasers (ECLs, linewidth $10 \mathrm{kHz}$ ) and another for the 32 extra channels carried by distributed feedback lasers (DFBs). The ECLs are placed on channel indices $\{1,7,13$, $18,23,28,34,40\}$. Delay-and-add polarization emulation is applied, the spectrum is flattened by an optical filter and sent on the channel.

The link consists of 2 erbium doped fiber amplifier (EDFA) spans of $100 \mathrm{~km}$ and $40 \mathrm{~km}$, respectively, for an extended interDCI distance of $140 \mathrm{~km}$ [14]. The launch power is set to 18 $\mathrm{dBm}$ total. At the end of the transmission link, the received WDM signal is band-pass filtered to select the channel of interest, amplified and fed to the coherent receiver frontend. The signal is down-converted using a local oscillator $(10-\mathrm{kHz}$ ECL). A digital storage oscilloscope with $80 \mathrm{GSa} / \mathrm{s}$ sampling rate is used to capture the signal for offline processing, which is identical to that of [18].

\section{TRAnSMission Results}

Due to the wavelength dependence of the transceivers and the non-flat noise properties of the EDFAs, the performance is wavelength dependent. The SNR for the channels of interest is depicted in Fig. 1.b. A variation of up to $1.2 \mathrm{~dB}$ is seen, which demands a rate-adaptive system if spectral efficiency is targeted.

We performed the experimental characterization of rateadaptive CFEC with various CM (BICM \& MLC) schemes and (16 \& 64QAM) modulation formats. The BER performance of the worst and the best WDM channels having various CM schemes is depicted in Fig. 2. For 16QAM, the BER

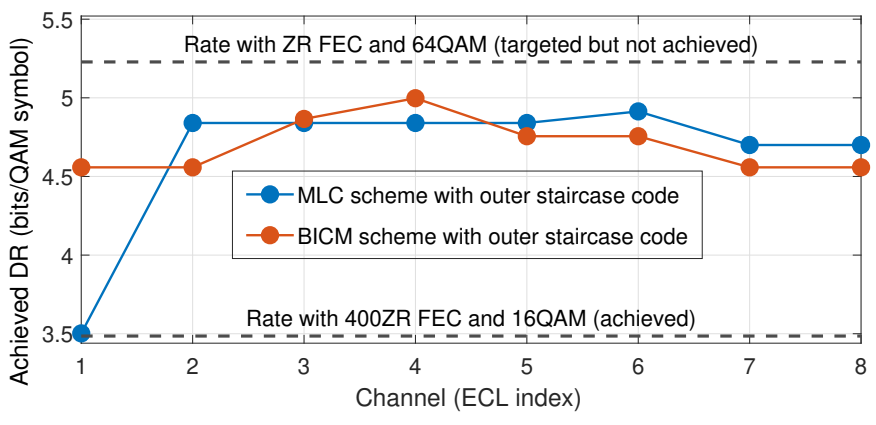

Fig. 3. Experimental maximum achieved data rate (DR) (bits/QAM symbol) of the studied coded-modulation (MLC \& BICM) schemes as a function of the channel given by external cavity laser (ECL) index.

performance is only calculated at the 400ZR data rate of 3.49 (bits/QAM symbol). For 64QAM, we measured the BER performance of BICM- and MLC-based polar-staircase codes for the data rate in the range of 3.99 to 5.30 (bits/QAM symbol) and in the range of 4.70 to 5.34 (bits/QAM symbol), respectively. Data rate is controlled by varying the values of $L_{0}$ and $K_{p c}$ bits. The ZR FEC with 64QAM and at data rate of 5.23 (bits/QAM symbol) is also targeted but cannot be achieved error free, as illustrated in Fig. 2. Similar to [7], the MLC has a slight performance advantage over BICM at the higher SNR channels, while also reducing the complexity.

For each ECL channel, we calculated the BER over the range of data rates and chose the maximum achieved data rate for which the measured BER is below the threshold $p$ of the outer HD code. The MLC and BICM results are given in Fig. 3. In all cases, 64QAM is considered except for the ECL index of 1 with MLC, where the system is unable to bring BER below the threshold of $p$. In that case, the system has to fall back to 16QAM and at 400ZR code rate.

The upper envelope, i.e., the maximum values, of the $\mathrm{CM}$ performance curves of Fig. 3 is used to maximize the achieved data rate for each WDM channel. The resulting optimal choice of the CM scheme for each channel is given in Table I together with the corresponding parameters $L_{0}$ and $K_{p c}$. Due to the varying channel conditions and too coarse granularity, the ZR FEC can not support the 64QAM code rate but has to fall back to 16QAM, resulting in a sum-rate of $1787 \mathrm{Gbps}$. The proposed scheme offers fine granularity and matches the channel conditions, achieving $37.6 \%$ increase in the sum-rate (2459 Gbps).

Following [11], we evaluate the requirements on the length of the interleaver $\pi_{1}$ (Fig. 4) in terms of the probability mass function (PMF) of the number of input bit errors per block of length 1022, which is the length of the $\mathrm{BCH}$ component codewords of the outer HD staircase code. The experimental data from Channel index 6 in Table I is exemplified, together with an AWGN simulation with the same MLC parameters and the theoretical independent distribution of errors as calculated in [11, Eq. (4)] for an average BER of $p=4.0 \times 10^{-3}$. An interleaver of length $\approx 50 \mathrm{BCH}$ codewords $(50 \times 1022$ bits) is required in the simulation to approach the theoretical distribution. In the experiment, longer interleaver is required of $\approx 150 \mathrm{BCH}$ codewords, which is mainly attributed to the 
TABLE I

PARAMETERS FOR THE UPPER ENVELOPE OF THE CM CURVES OF FIG. 3. $T_{r e d}$ DENOTES REDUCTION IN THE NUMBER OF INNER SD DECODINGS AT A GIVEN RECEIVER THROUGHPUT, COMPARED TO BICM.

\begin{tabular}{|c|c|c|c|c|c|}
\hline $\begin{array}{c}\text { ECL } \\
\text { index }\end{array}$ & $\begin{array}{c}\text { CM } \\
\text { scheme }\end{array}$ & $K_{p c}$ & $L_{0}$ & $T_{\text {red }}(\%)$ & $\begin{array}{c}\text { Throughput } \\
(\mathrm{Gb} / \mathrm{s})\end{array}$ \\
\hline 1 & BICM & 830 & 0 & 0 & 291.8 \\
\hline 2 & MLC & 846 & 254 & 23.09 & 309.8 \\
\hline 3 & BICM & 886 & 0 & 0 & 311.4 \\
\hline 4 & BICM & 902 & 0 & 0 & 319.8 \\
\hline 5 & MLC & 846 & 254 & 23.09 & 309.8 \\
\hline 6 & MLC & 846 & 386 & 31.33 & 314.5 \\
\hline 7 & MLC & 846 & 62 & 6.83 & 300.8 \\
\hline 8 & MLC & 846 & 62 & 6.83 & 300.8 \\
\hline
\end{tabular}

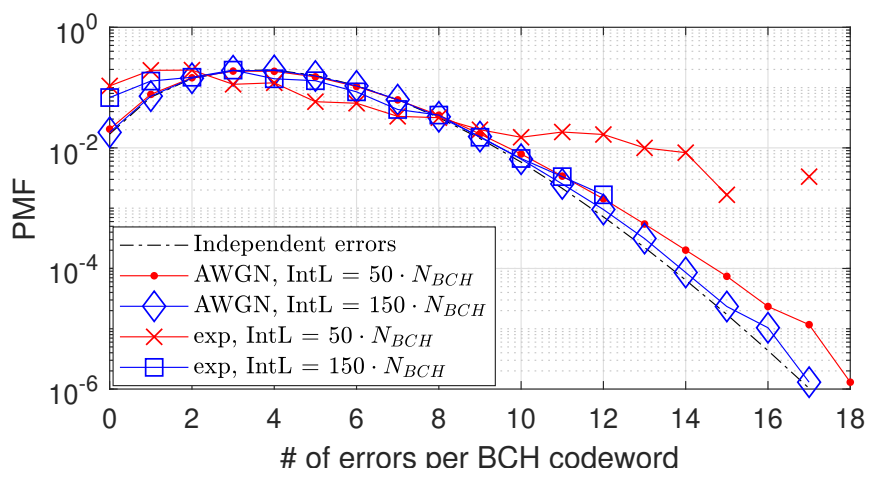

Fig. 4. PMF of the number of errors after $\pi_{1}^{-1}$ per BCH codeword (of length 1022) for different interleaver lenghts in experiment and an AWGN channel simulation for an MLC parameterized as for Channel 6 in Table I.

residual phase noise and imperfect DSP, which correlate the errors further. Such interleaver lengths are well within the capabilities of modern coherent technology (e.g. 172,032 bits employed in ZR+ [9]), and is in terms of latency negligible compared to that imposed by the staircase decoder.

\section{A. A note on decoding complexity}

As shown in Table I, extra complexity savings can be achieved by utilizing MLC instead of BICM. In this paper, MLC or BICM are selected based on performance, resulting in an average of $11.4 \%$ of complexity reduction in terms of decodings (2). As shown in [19], at the cost of slight performance degradation, the complexity reductions can be increased up to $70 \%$ when the MLC is parameterized for that target.

\section{CONCLUSIONS}

Error-free transmission, based on evaluating BER and distribution of errors per BCH block into the HD outer decoder, is demonstrated by employing a concatenated coding scheme having rate-adaptivity with one and two degrees of freedom in an experimental $140 \mathrm{~km}$ fibre-optic link. A fixed FEC rate system like ZR must choose between 16QAM and 64QAM. The former does not utilize the available varying SNR per channel, while the latter is unachievable error-free. In contrast, our proposed scheme offers near-continuous granularity and matches the channel conditions, achieving higher data rates.
The experimental demonstration suggests that the proposed MLC scheme can be used as a flexible and controllable FEC solution for future optical networks and ZR extensions (ZR+).

\section{ACKNOWLEDGMENT}

This work was supported by the Innovation Fund Denmark (INCOM project No. 8057-00059B) and DNRF Research Center of Excellence, SPOC-I, ref. DNRF123. The authors would like to thank Pawel M. Kaminski for his help in setting up the experimental testbed.

\section{REFERENCES}

[1] L. Paraschis and K. Raj, "Innovations in DCI transport networks," in Optical Fiber Telecommunications VII, pp. 673-718, Elsevier, 2020.

[2] A. Bisplinghoff, S. Langenbach, and T. Kupfer, "Low-power, phaseslip tolerant, multilevel coding for M-QAM,' Journal of Lightwave Technology, vol. 35, no. 4, pp. 1006-1014, 2016.

[3] M. Barakatain and F. R. Kschischang, "Low-complexity concatenated LDPC-staircase codes," Journal of Lightwave Technology, vol. 36 , no. 12, pp. 2443-2449, 2018.

[4] Y. Koganei, T. Oyama, K. Sugitani, H. Nakashima, and T. Hoshida, "Multilevel coding with spatially coupled repeat-accumulate codes for high-order QAM optical transmission," Journal of Lightwave Technology, vol. 37, no. 2, pp. 486-492, 2019.

[5] T. Mehmood, M. P. Yankov, A. Fisker, K. Gormsen, and S. Forchhammer, "Rate-adaptive concatenated polar-staircase codes for data center interconnects," in Optical Fiber Communication Conference, pp. Th1I6, Optical Society of America, 2020.

[6] M. Barakatain and F. R. Kschischang, "Low-complexity rate- and channel-configurable concatenated codes," Journal of Lightwave Technology, vol. 39, no. 7, pp. 1976-1983, 2021.

[7] T. Mehmood, M. P. Yankov, S. Iqbal, and S. Forchhammer, "Flexible multilevel coding with concatenated polar-staircase codes for m-qam," IEEE Transactions on Communications, vol. 69, no. 2, pp. 728-739, 2020.

[8] B. S. G. Pillai, B. Sedighi, K. Guan, N. P. Anthapadmanabhan, W. Shieh, K. J. Hinton, and R. S. Tucker, "End-to-end energy modeling and analysis of long-haul coherent transmission systems," Journal of Lightwave Technology, vol. 32, no. 18, pp. 3093-3111, 2014.

[9] "Implementation agreement 400ZR," in Optical Internetworking Forum 0.10-Draft, 2018.

[10] B. Smith, I. Lyubomirsky, and S. Bhoja, "Leveraging 400G ZR FEC technology," in IEEE 802.3 Beyond $10 \mathrm{~km}$ Optical PHYs Study Group, 2017.

[11] Y. Cai, W. Wang, W. Qian, J. Xing, K. Tao, J. Yin, S. Zhang, M. Lei, E. Sun, H.-C. Chien, et al., "FPGA investigation on error-flare performance of a concatenated staircase and Hamming FEC code for 400G inter-data center interconnect," Journal of Lightwave Technology, vol. 37 , no. 1, pp. 188-195, 2019.

[12] L. Schmalen, L. M. Zhang, and U. Gebhard, "Distributed rate-adaptive staircase codes for connectionless optical metro networks," in 2017 Optical Fiber Communications Conference, pp. 1-3, IEEE, 2017.

[13] A. e. Morello, "DVB-S2: The second generation standard for satellite broad-band services," Proceedings of the IEEE, vol. 94, no. 1, 2006.

[14] P. Bower, "What is ZR+?," in Ciena, May 2021.

[15] "OTU4 long-reach interface," in document ITU-T Rec. G.709.2/Y.1331.2, ITU, July 2018.

[16] M. P. Yankov, H. E. Hansen, F. Da Ros, P. M. Kaminski, E. P. da Silva, M. Galili, L. K. Oxenløwe, and S. Forchhammer, "Probabilistic shaping for the optical phase conjugation channel," IEEE Journal of Selected Topics in Quantum Electronics, vol. 27, no. 2, pp. 1-16, 2020.

[17] A. Alvarado, E. Agrell, D. Lavery, R. Maher, and P. Bayvel, "Replacing the soft-decision FEC limit paradigm in the design of optical communication systems," Journal of Lightwave Technology, vol. 33, no. 20, pp. 4338-4352, 2015.

[18] M. P. Yankov, P. M. Kaminski, H. E. Hansen, and F. Da Ros, "SNR optimization of multi-span fiber optic communication systems employing EDFAs with non-flat gain and noise figure," Journal of Lightwave Technology, pp. 1-1, 2021.

[19] T. Mehmood, M. P. Yankov, and S. Forchhammer, "Rate-adaptive concatenated multilevel coding with fixed decoding complexity," in International Symposium on Topics in Coding (ISTC), pp. 1-5, IEEE, 2021. 